\title{
High-Normal Serum Thyrotropin Levels Increased the Risk of Non-Alcoholic Fatty Liver Disease in Euthyroid Subjects with Type 2 Diabetes
}

\author{
Ying $\operatorname{Tan}^{1, *}$ \\ Xixiang Tang ${ }^{1,2, *}$ \\ Panwei Mu' \\ Yi Yang' \\ Mei Li ${ }^{1,2}$ \\ Yuanpeng $\mathrm{Nie}^{1}$ \\ Haicheng $\mathrm{Li}^{\prime}$ \\ Yanhua Zhu (D) \\ Yanming Chen' \\ 'Department of Endocrinology \& \\ Metabolism, The Third Affiliated Hospital \\ of Sun Yat-sen University, Guangdong \\ Provincial Key Laboratory of Diabetology, \\ Guangzhou, People's Republic of China; \\ ${ }^{2}$ VIP medical service center, The Third \\ Affiliated Hospital of Sun Yat-sen \\ University, Guangzhou, People's Republic \\ of China
}

*These authors contributed equally to this work

\begin{abstract}
Purpose: The aim of this study was to investigate the association between high-normal thyrotropin (TSH) levels and the prevalence of non-alcoholic fatty liver disease (NAFLD) in euthyroid patients with T2DM.
\end{abstract}

Methods: A total of 2289 euthyroid adults with T2DM were included in this cross-sectional study conducted at the Third Affiliated Hospital of Sun Yat-sen University from January 2016 to December 2018. NAFLD was diagnosed by abdominal ultrasound. Thyroid function parameters, including the levels of TSH, free triiodothyronine (FT3) and free thyroxine (FT4), were analyzed. The patients were stratified by quartiles (Q1-4) of TSH levels. Multivariate logistic regression models were used to evaluate the association between the quartiles of TSH levels and the risk of NAFLD in euthyroid adults with T2DM.

Results: There were 940 (41.1\%) euthyroid adults with T2DM who were diagnosed with NAFLD. The subjects were divided according to the thyroid function parameter quartiles. The prevalence of NAFLD increased with increasing TSH level quartiles (Q1 to Q4: 34.8\%, $37.5 \%, 44.9 \%$ and $47.0 \%, P<0.01)$ but not with increasing FT3 or FT4 level quartiles. In the multivariate logistic regression model, compared with the lowest TSH level quartile (Q1), the highest TSH level quartile (Q4) $(\mathrm{OR}=1.610,95 \% \mathrm{CI}=1.131-2.289, P=0.008)$ was independently associated with an increased risk of NAFLD in euthyroid adults with T2DM after adjusting for multiple confounders. After additional stratification by the level of glycosylated haemoglobin (HbAlc) and body mass index (BMI), the highest TSH level quartile was still independently associated with an increased risk of NAFLD in euthyroid patients with T2DM who had an $\mathrm{HbA} 1 \mathrm{c}$ level $\geq 7 \%$ or a $\mathrm{BMI}<28 \mathrm{~kg} / \mathrm{m}^{2}$.

Conclusion: High-normal serum TSH levels are significantly associated with the presence of NAFLD in T2DM patients with euthyroid function, which provide novel insight for treating NAFLD.

Keywords: non-alcoholic fatty liver disease, type 2 diabetes, thyroid hormone

\section{Introduction}

Non-alcoholic fatty liver disease (NAFLD) is emerging as a public health issue worldwide and encompasses a wide spectrum of pathologic liver conditions ranging from simple steatosis to steatohepatitis and cirrhosis. ${ }^{1,2}$ NAFLD and type 2 diabetes mellitus (T2DM) are common conditions that regularly coexist. The overall prevalence of NAFLD among patients with T2DM is $55.5 \% .{ }^{3}$ These two diseases could act synergistically and drive many adverse outcomes, including the complications of diabetes, cardiovascular disease, liver-related mortality and all-cause 
mortality. ${ }^{4,5}$ Thus, a better understanding of the risk factors for the incidence of NAFLD in patients with T2DM is needed.

It is well known that thyroid hormone regulates favorable metabolic processes, including thermogenesis, lipid metabolism, and carbohydrate metabolism. ${ }^{6}$ Thyroid hormone is involved in lipid metabolism in hepatocytes. ${ }^{7}$ Abundant studies have shown that both hypothyroidism and subclinical hypothyroidism are associated with NAFLD.${ }^{8,9}$ Several studies have demonstrated that thyroid hormone is associated with the risk of NAFLD in euthyroid subjects. ${ }^{10-12}$ However, few data exist regarding the association between serum thyroid hormone levels in the normal range and NAFLD in patients with T2DM.

Therefore, this study aimed to investigate the association between normal thyroid function and the prevalence of NAFLD in euthyroid adults with T2DM.

\section{Methods}

\section{Study Design and Participants}

This retrospective cross-sectional study was conducted in our hospital between January 2016 and December 2018. T2DM patients who were admitted to the hospital for glycemic control were enrolled. T2DM was diagnosed according to the 1999 criteria of the World Health Organization (WHO). ${ }^{13}$ Subjects who had pairs of thyroid function tests and hepatic ultrasonography measurements were included in the study. Only euthyroid subjects participated in the present study. In this study, euthyroidism was defined as a TSH level between 0.35 and $4.94 \mu \mathrm{IU} /$ $\mathrm{mL}$, FT4 level between 9.01 and $19.05 \mathrm{pmol} / \mathrm{L}$ and FT3 level between 2.63 and $5.70 \mathrm{pmol} / \mathrm{L}$ according to the normal reference value of thyroid function tests in our hospital. The diagnosis of NAFLD was made based on the guidelines for prevention and treatment of NAFLD. ${ }^{14}$ The exclusion criteria were as follows: (1) type 1 diabetes, latent immune diabetes of adults, and specific types of diabetes; (2) history of thyroid diseases, abnormal thyroid hormone levels, treatment history with anti-thyroid drugs or other drugs that could potentially influence thyroid function (eg, amiodarone, lithium, corticosteroids, etc.); (3) subjects reporting significant alcohol consumption ( $>30 \mathrm{~g} /$ day for men and $>20 \mathrm{~g} /$ day for women); and (5) subjects infected with hepatitis B or hepatitis C virus, known to have pre-existing chronic liver disease (eg, Wilson's disease, haemochromatosis, autoimmune hepatitis, primary biliary cholangitis, primary sclerosing cholangitis, drug-induced liver disease). Ethics approval was obtained from the Third Affiliated Hospital of Sun Yat-sen University Network Ethics Committee. Informed consent was obtained from all participants.

\section{Data Collection}

Demographic and clinical information, including age, sex, height, body weight, blood pressure, lifestyle factors (smoking status and alcohol consumption), comorbidities, duration of diabetes, diabetes therapy (antihyperglycemic agents, insulin injection) and medications, was collected. Concentrations of biochemical parameters, including fasting plasma glucose (FPG), blood urea nitrogen (BUN), creatinine, uric acid, triglycerides, total cholesterol, highdensity lipoprotein cholesterol (HDL-C), low-density lipoprotein cholesterol (LDL-C) plasma aspartate transaminase (AST), alanine transaminase (ALT), gamma glutamyltransferase (GGT), alkaline phosphatase (ALP), total bilirubin (TBIL) and indirect bilirubin (IBIL), were measured by a HITACHI 7180 automatic analyzer. $\mathrm{HbAlc}$ was measured by the D-10 haemoglobin testing program (Bio-Rad) with high-performance liquid chromatography (HPLC). Thyroid function tests, including free thyroxine (FT4), free triiodothyronine (FT3) and thyroidstimulating hormone (TSH), were measured by chemiluminescent immunoassays on an Abbott i4000 automatic analyzer. The homoeostasis model assessment of insulin resistance (HOMA-IR) was calculated according to the formula fasting insulin*FPG/22.5. ${ }^{15}$

\section{Diagnosis of NAFLD}

NAFLD is defined as the presence of steatosis in the liver when secondary causes of fatty infiltration in the liver have been excluded. ${ }^{14}$ Hepatic steatosis was diagnosed using ultrasound scans according to the guidelines for prevention and treatment of non-alcoholic fatty liver disease. ${ }^{14}$ The ultrasound examinations were performed with GE-LOGIQ E9 ultrasound machines by experienced ultrasonographic physicians who were blinded to the study.

\section{Statistical Analysis}

Database management and statistical analysis were performed by using SPSS 22.0 for Windows (SPPS Inc, Chicago, IL, USA). Descriptive statistics are presented as the mean \pm standard deviation for continuous variables or as numbers and percentages for categorical variables. All patients were grouped into quartiles of TSH (Q1 = the first quartile; Q2 = 
the second quartile; Q3 = the third quartile; $\mathrm{Q} 4$ = the fourth quartile). Continuous variables were compared by analysis of variance (ANOVA), while categorical variables were compared using the Pearson chi square test. Multivariate logistic regression analysis was performed to evaluate the effect of TSH levels on the risk of NAFLD in T2DM patients. To further assess whether glycemic status and obesity status confound the relationship between TSH and the risk of NAFLD, subgroup analyses were performed based on $\mathrm{HbAlc}$ levels ( $<$ $7.0 \%$ and $\geq 7.0 \%)$ and BMI $\left(<28\right.$ and $\left.\geq 28 \mathrm{~kg} / \mathrm{m}^{2}\right)$. A 2-tailed $P$ value $<0.05$ was considered indicative of statistical significance.

\section{Results}

From January 2016 to December 2018, 6109 patients with T2DM were enrolled in the study. A total of 2734 patients who did not finish thyroid function testing or hepatic ultrasonography measurements were excluded. Another 1086 patients with abnormal thyroid function were also excluded. Finally, 2289 patients (1247 men and 1042 women; mean age, $55.8 \pm 14.4$ years) were included in the analysis (Figure 1). The median diabetes duration of the patients was $6(2,11)$ years, with a mean $\mathrm{HbAlc}$ level of $9.04 \pm$ $2.52 \%$. The mean FPG was $10.15 \pm 6.45 \mathrm{mmol} / \mathrm{L}$.

A total of 940 participants with T2DM were diagnosed with NAFLD, and the total prevalence of NAFLD in subjects with T2DM was $41.1 \%$. As shown in Supplemental Table 1, patients with T2DM and NAFLD showed an elevated level of TSH, BUN, UA, TC, TG,
HbA1c, BMI, SDP and DBP, with a lower diabetic duration, HDL-C level (all $P<0.05$ ). There were also significant differences in proportion of insulin injection, sulfonylureas, metformin, thiazolidinediones, GLP-1 receptor agonists, SGLT-2 inhibitors, statin and comorbidity of hyperlipidaemia between the type 2 diabetic patients with and without NAFLD (all $P<0.05$ ).

Then as shown in Figure 2, the prevalence of NAFLD had a positive trend with increasing quartile levels of TSH (34.8\%, 37.5\%, 44.9\% and 47.0\%, Q1 to Q4, respectively, $P<0.05$ ) but not with FT3 or FT4 quartile levels. Therefore, we divided the participants according to the quartiles of TSH level. Table 1 shows the clinical characteristics of the subjects grouped according to the quartiles of TSH levels. The concentrations of TSH were $<0.96 \mathrm{mIU} / \mathrm{L}$ in Q1 subjects, $0.96-1.44 \mathrm{mIU} / \mathrm{L}$ in Q2 subjects, $1.44-2.13 \mathrm{mIU} / \mathrm{L}$ in Q3 subjects, and $>2.13 \mathrm{mIU} / \mathrm{L}$ in $\mathrm{Q} 4$ subjects. Age, diabetes duration, proportion of insulin injection, metformin intake, comorbidity of hyperlipidaemia and hypertension, and levels of ALT, TBIL, Cr, BUN, TG, FPG, HbA1c, and FT3 varied within groups $(P<0.05)$. No significant differences in the other parameters were observed among subjects with different TSH quartiles.

\section{Univariate and Multivariate Logistic Regression Analysis}

In the univariable model, the TSH levels in Q3 and Q4 showed significant associations with the risk of NAFLD $(P<0.0001)$ (Supplemental Table 2). When further adjusting

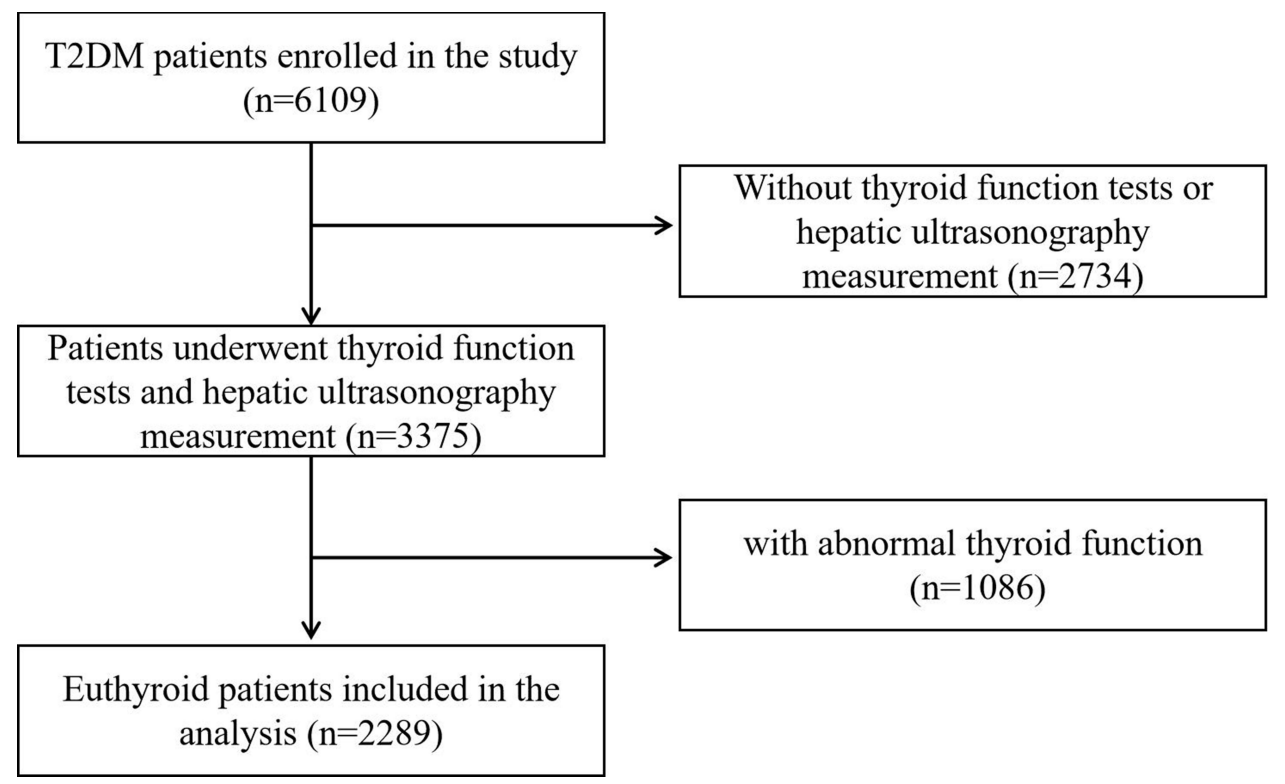

Figure I Flowchat of the study. 


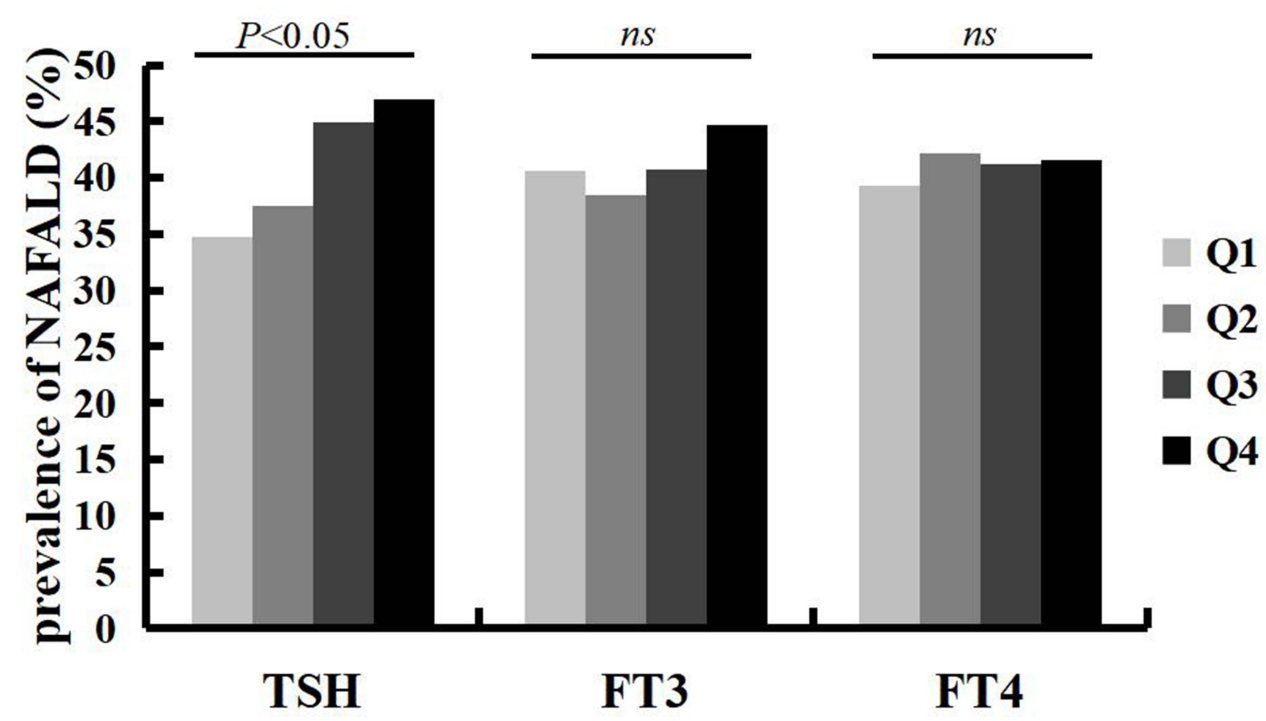

Figure 2 Prevalence of non-alcoholic fatty liver disease (NAFLD) according to thyroid function in the present study.

for age and sex (model 1), the TSH levels of participants in Q3 and Q4 were still independently related to the risk of NAFLD $(P<0.05)$. After further adjustment for BMI, diabetes duration, levels of HDL-C, LDL-C, TGs, HbA1c, FT3, and FT4, smoking status, alcohol intake, insulin injection and metformin use, patients in Q4 remained at a higher risk of NAFLD than patients in Q1 $(P<0.05)$ (model 2) (Figure 3).

\section{Subgroup Analysis}

As shown in Supplemental Table 3, TSH levels of the patients in the Q3 and Q4 quartiles were significantly associated with the risk of NAFLD regardless of the HbA1c level and in the BMI $<28 \mathrm{~kg} / \mathrm{m}^{2}$ subgroup $(P<0.05)$ in the univariable model or after further adjustment for age and sex (model 1). In the multivariate analysis adjusted for age, sex, BMI, diabetes duration, levels of HDL-C, LDL-C, TGs, HbA1c, FT3, and FT4, smoking status, alcohol intake, insulin injection and metformin use, TSH levels in Q4 continued to be a strong determinant of the risk of NAFLD in the HbA1c $\geq 7.0 \%$ or BMI $<28 \mathrm{~kg} / \mathrm{m}^{2}$ subgroup (all $P<0.05$ ) (Figure 4).

\section{Discussion}

This cross-sectional study revealed that high-normal TSH levels were associated with an increased risk of NAFLD in euthyroid adults with T2DM, particularly for subjects with an $\mathrm{HbA} 1 \mathrm{c}$ level $\geq 7.0 \%$ or a $\mathrm{BMI}<28 \mathrm{~kg} / \mathrm{m}^{2}$. This result suggested that a high-normal TSH level was an independent risk factor for NAFLD in T2DM patients.
NAFLD is common among individuals with T2DM. ${ }^{16}$ The prevalence of NAFLD in T2DM patients differed by sex and the presence of obesity, hypertension, dyslipidemia, coronary heart disease, and chronic kidney disease. ${ }^{17-20}$ Thyroid hormones have important roles in regulating energy balance and metabolism. ${ }^{7}$ Some studies have explored whether thyroid hormone disorder is a risk factor for the development of NAFLD and T2DM. ${ }^{21-23}$ Several studies have shown that a low-normal thyroid hormone level is associated with high fasting glucose levels and a high HbAlc level. ${ }^{24}$ Furthermore, higher-normal serum TSH levels were associated with the incidence of T2DM. ${ }^{25}$ Regarding the relationship between TSH and NAFLD, many studies have proven that subclinical hypothyroidism is a risk factor for NAFLD, and an increase in TSH levels is positively correlated with the incidence of NAFLD. ${ }^{26,27}$ Notably, high TSH levels have been reported to be associated with the severity of hepatic steatosis. ${ }^{28}$ In addition, in euthyroid subjects, several studies have demonstrated that high TSH levels are associated with the risk of NAFLD. ${ }^{11,28}$ Recently, a study with small participant sample showed that NAFLD in euthyroid T2DM patients may be associated with thyroid hormone resistance-like manifestation. ${ }^{29}$ Consistently, our study confirmed that a higher-normal TSH level was associated with an increased risk of NAFLD in euthyroid subjects with T2DM.

It is likely that TSH plays an important role in the incidence of NAFLD in patients with T2DM. Of note, the present study revealed that serum TSH levels in the upper normal range are associated with the incidence of NAFLD 
Table I Clinical Characteristics of the Participants According to Quartiles of Serum TSH Levels

\begin{tabular}{|c|c|c|c|c|c|c|}
\hline \multirow[t]{2}{*}{ Variables $(\%$ or Mean \pm SD) } & \multirow[t]{2}{*}{ Total } & \multicolumn{4}{|c|}{ Quartile of TSH } & \multirow[t]{2}{*}{$P$-value } \\
\hline & & I & 2 & 3 & 4 & \\
\hline $\mathrm{n}$ & 2289 & 572 & 573 & 572 & 572 & \\
\hline $\mathrm{TSH}, \mathrm{mlU} / \mathrm{L}$ & $1.65 \pm 0.91$ & $0.71 \pm 0.16$ & $1.20 \pm 0.14$ & $1.76 \pm 0.20$ & $2.93 \pm 0.68$ & $<0.001$ \\
\hline Male, n (\%) & I 247 (54.5) & $351(61.4)$ & $326(56.9)$ & $307(53.7)$ & $263(46.0)$ & $<0.001$ \\
\hline Age, years & $55.8 \pm 14.4$ & $55.1 \pm 13.8$ & $54.5 \pm 14.8$ & $56.2 \pm 14.7$ & $57.6 \pm 14.3$ & 0.002 \\
\hline Waist circumference, $\mathrm{cm}$ & $89.4 \pm 10.5$ & $89.23 \pm 10.23$ & $88.46 \pm 10.77$ & $90.14 \pm 10.49$ & $89.82 \pm 10.28$ & 0.196 \\
\hline BMI, kg/m2 & $24.69 \pm 8.93$ & $24.16 \pm 3.82$ & $24.53 \pm 10.12$ & $25.18 \pm 9.52$ & $24.87 \pm 10.49$ & 0.319 \\
\hline $\mathrm{SBP}, \mathrm{mmHg}$ & $|3| .3 \pm 20.4$ & $129.9 \pm 19.9$ & $130.4 \pm 19.3$ & $132.4 \pm 21.6$ & $132.4 \pm 20.7$ & 0.131 \\
\hline $\mathrm{DBP}, \mathrm{mmHg}$ & $79.3 \pm 11.7$ & $79.5 \pm 12.2$ & $79.7 \pm 11.1$ & $79.9 \pm 12.0$ & $78.1 \pm 11.4$ & 0.082 \\
\hline \multicolumn{7}{|l|}{ Blood biochemical indices } \\
\hline ALT, U/I & $28.6 \pm 5.1$ & $30.9 \pm 8.7$ & $26.5 \pm 5.5$ & $24.7 \pm 8.4$ & $28.6 \pm 5.0$ & 0.012 \\
\hline AST, U/I & $24.3 \pm 6.1$ & $25.3 \pm 9.2$ & $22.4 \pm 4.1$ & $26.7 \pm 3.7$ & $22.7 \pm 5.6$ & 0.054 \\
\hline TBIL, nmol/L & $11.46 \pm 5.64$ & $11.89 \pm 6.40$ & $|1.66 \pm 5.4|$ & $11.38 \pm 5.14$ & $10.90 \pm 5.51$ & 0.026 \\
\hline IBIL, nmol/L & $7.50 \pm 3.63$ & $7.68 \pm 3.87$ & $7.67 \pm 3.63$ & $7.47 \pm 3.47$ & $7.18 \pm 3.52$ & 0.088 \\
\hline GGT, nmol/L & $25(17,38)$ & $23(16,37)$ & $25(18,37)$ & $26(18,40)$ & $24(16,37.5)$ & 0.506 \\
\hline ALP, nmol/L & $77.24 \pm 37.22$ & $78.37 \pm 35.68$ & $77.18 \pm 37.75$ & $76.28 \pm 33.23$ & $77.15 \pm 41.76$ & 0.836 \\
\hline $\mathrm{Cr}, \mathrm{umol} / \mathrm{l}$ & $81.10 \pm 61.41$ & $76.52 \pm 45.43$ & $75.42 \pm 39.51$ & $83.67 \pm 67.29$ & $88.86 \pm 82.80$ & $<0.001$ \\
\hline BUN, umol/l & $5.93 \pm 2.74$ & $5.79 \pm 2.65$ & $5.63 \pm 2.02$ & $6.05 \pm 3.02$ & $6.25 \pm 3.09$ & 0.001 \\
\hline UA, umol/l & $366.95 \pm 110.59$ & $358.88 \pm 110.95$ & $363.67 \pm 103.27$ & $375.77 \pm|1| 1.5 \mid$ & $369.59 \pm 115.81$ & 0.065 \\
\hline $\mathrm{TC}, \mathrm{mmol} / \mathrm{l}$ & $4.79 \pm 1.32$ & $4.74 \pm 1.36$ & $4.78 \pm 1.17$ & $4.83 \pm 1.23$ & $4.82 \pm 1.48$ & 0.619 \\
\hline $\mathrm{TG}, \mathrm{mmol} / \mathrm{l}$ & $1.34(0.94,1.99)$ & $1.20(0.86,1.80)$ & $1.35(0.93,1.96)$ & I.4I $(0.99,2.07)$ & $1.41(0.96,2.11)$ & 0.049 \\
\hline $\mathrm{HDL}-\mathrm{C}, \mathrm{mmol} / \mathrm{l}$ & $1.11 \pm 0.32$ & $1.10 \pm 0.33$ & $1.11 \pm 0.32$ & $I . I I \pm 0.31$ & $1.12 \pm 0.32$ & 0.827 \\
\hline LDL-C, mmol/l & $2.94 \pm 0.99$ & $2.94 \pm 0.98$ & $2.98 \pm 0.96$ & $2.98 \pm 0.96$ & $2.86 \pm 1.05$ & 0.168 \\
\hline \multicolumn{7}{|l|}{ Lifestyles, n (\%) } \\
\hline Smoking, n (\%) & $586(25.6)$ & $178(3||)$. & $170(29.7)$ & $132(23.1)$ & $106(18.5)$ & $<0.001$ \\
\hline Alcohol, n (\%) & $347(15.2)$ & $89(15.6)$ & $100(17.5)$ & $74(12.9)$ & $84(14.7)$ & 0.185 \\
\hline \multicolumn{7}{|l|}{ Diabetes-related variables } \\
\hline Diabetes duration, years & $6(2,11)$ & $6(1,11)$ & $6(2,10)$ & $6(2,11)$ & $8(2,14)$ & $<0.001$ \\
\hline $\mathrm{FPG}, \mathrm{mmol} / \mathrm{L}$ & $10.15 \pm 6.45$ & $11.42 \pm 7.36$ & $10.03 \pm 6.40$ & $9.81 \pm 6.15$ & $9.35 \pm 5.58$ & $<0.001$ \\
\hline $\mathrm{HbAlc}, \%$ & $9.04 \pm 2.52$ & $9.67 \pm 2.70$ & $9.09 \pm 2.51$ & $8.88 \pm 2.41$ & $8.54 \pm 2.30$ & $<0.001$ \\
\hline HOMA-IR & $6.01 \pm 17.74$ & $5.98 \pm 12.84$ & $5.30 \pm 8.23$ & $7.47 \pm 31.80$ & $5.32 \pm 6.18$ & 0.405 \\
\hline FT3, pmol/l & $4.32 \pm 0.67$ & $4.25 \pm 0.67$ & $4.37 \pm 0.65$ & $4.34 \pm 0.67$ & $4.34 \pm 0.68$ & 0.018 \\
\hline $\mathrm{FT} 4, \mathrm{pmol} / \mathrm{l}$ & $15.44 \pm 2.18$ & $15.42 \pm 2.19$ & $15.55 \pm 2.14$ & $15.46 \pm 2.22$ & $15.33 \pm 2.18$ & 0.414 \\
\hline \multicolumn{7}{|l|}{ Medications, $\mathbf{n}(\%)$} \\
\hline Insulin injection, $\mathrm{n}(\%)$ & $839(36.7)$ & $24 I(42.1)$ & $212(37.0)$ & $193(33.7)$ & $193(33.7)$ & 0.009 \\
\hline Sulfonylureas & $572(25.0)$ & $147(25.7)$ & $147(25.7)$ & $|4|(24.7)$ & $137(24.0)$ & 0.884 \\
\hline Glinides & $80(3.5)$ & $20(3.5)$ & $14(2.4)$ & $20(3.5)$ & $26(4.5)$ & 0.290 \\
\hline Metformin & $1340(58.5)$ & $356(62.2)$ & $343(59.9)$ & $348(60.8)$ & $293(51.2)$ & 0.001 \\
\hline Thiazolidinediones & $56(2.4)$ & $19(3.3)$ & $12(2.1)$ & $16(2.8)$ & $9(1.6)$ & 0.235 \\
\hline Glucosidase inhibitor & $74 \mid(32.4)$ & $205(35.8)$ & $173(30.2)$ & $178(3||)$. & $185(32.3)$ & 0.187 \\
\hline DPP-4 Inhibitors & $475(20.8)$ & $107(18.7)$ & $120(20.9)$ & $|2|(2 \mid .2)$ & $127(22.2)$ & 0.521 \\
\hline GLP-I receptor agonists & $45(2.0)$ & $9(1.6)$ & $9(1.6)$ & $13(2.3)$ & $14(2.4)$ & 0.596 \\
\hline SGLT2 Inhibitors & $37(1.6)$ & $7(1.2)$ & $14(2.4)$ & $9(1.6)$ & $7(1.2)$ & 0.311 \\
\hline Statin & | $486(64.9)$ & $354(61.9)$ & $374(65.3)$ & $373(65.2)$ & $385(67.3)$ & 0.285 \\
\hline Anti-platelet & $1005(43.9)$ & $243(42.5)$ & $240(41.9)$ & $246(43.0)$ & $276(48.3)$ & 0.112 \\
\hline
\end{tabular}

(Continued) 
Table I (Continued).

\begin{tabular}{|l|c|c|c|c|c|c|}
\hline \multirow{2}{*}{ Variables (\% or Mean \pm SD) } & \multirow{2}{*}{ Total } & \multicolumn{4}{|c|}{ Quartile of TSH } & \multirow{2}{*}{ P-value } \\
\cline { 3 - 6 } & & $\mathbf{I}$ & $\mathbf{2}$ & $\mathbf{3}$ & \\
\hline Comorbidity, n (\%) & & & & & \\
Cardiovascular disease & $194(8.5)$ & $50(8.7)$ & $45(7.9)$ & $47(8.2)$ & $52(9.1)$ & 0.881 \\
Hyperlipidemia & $115(5.0)$ & $23(4.0)$ & $18(3.1)$ & $35(6.1)$ & $39(6.8)$ & 0.013 \\
Hypertension & $799(34.9)$ & $185(32.3)$ & $180(31.4)$ & $205(35.8)$ & $229(40.0)$ & 0.009 \\
\hline
\end{tabular}

Note: Data are mean $\pm S D$, median (25th to 75 th percentile) or $n(\%)$.

Abbreviations: TSH, thyroid-stimulating hormone; BMI, body mass index; SBP, systolic blood pressure; DBP, diastolic blood pressure; FPG, fasting plasma glucose; HOMAIR, homeostatic model assessment of insulin resistance; FT3, free triiodothyronine; FT4, free thyroxine; ALT, alanine aminotransferase; AST, aspartate aminotransferase; TBIL, total bilirubin; IBIL, indirect bilirubin; GGT, glutamyl transpeptidase; ALP, alkaline phosphatase; Cr, plasma creatinine; BUN, blood urea nitrogen; UA, uric acid; TC, total cholesterol; TG, triglycerides; HDL-C, high density lipoprotein cholesterol; LDL-C, low density lipoprotein cholesterol.

in T2DM patients with $\mathrm{HbA} 1 \mathrm{c} \geq 7 \%$ but not in patients with $\mathrm{HbA} 1 \mathrm{c}<7 \%$. A longitudinal study demonstrated that an increase in TSH levels was related to an increase in HbA1c and FPG levels and that there was an increased risk of NAFLD with increasing levels of HbAlc independent of obesity. ${ }^{30}$ In addition, a high-normal TSH level is related to increased insulin resistance and increased glycemic variability and may contribute to glycemic disorders in diabetes. ${ }^{24,31}$ Therefore, a high-normal serum TSH level remains a significant additional risk factor for the increased risk of NAFLD in type 2 diabetic patients with poor glycemic control. In patients with T2DM without evidence of thyroid disease, a higher serum TSH level was closely associated with central obesity and hyperlipidaemia, which are closely related to the pathogenesis of NAFLD. ${ }^{32}$ Interestingly, the present study found that a high-normal TSH level is a risk factor for the incidence of NAFLD in non-obese patients with T2DM but not in obese patients. The major contributing factors to lean NAFLD have been reported and include insulin resistance, hypertriglyceridaemia, and hyperandrogenaemia, which may be associated with TSH levels.

The mechanism for the association between a high-normal serum TSH level and an increased risk of NAFLD remains unclear. However, several possible mechanisms are available to explain the link. The relationship between NAFLD and T2DM has been well established, which could be explained by insulin resistance and compensatory hyperinsulinaemia leading to abnormal lipid metabolism and hepatic triglyceride accumulation in NAFLD. ${ }^{33}$ Hypothyroidism has been associated with insulin resistance, obesity, and even metabolic syndrome, which play an important role in the development of NAFLD. ${ }^{10}$ Moreover, in euthyroid individuals without T2DM or with T2DM, increased TSH-normal levels were associated with visceral obesity, increased triglyceride concentrations and reduced insulin sensitivity, which may promote the occurrence of NAFLD. ${ }^{25}$ In addition, NAFLD patients have displayed increased tumor necrosis factor alpha (TNF-a), elevated leptin levels, and decreased adiponectin levels compared to healthy controls. ${ }^{34,35}$ Alterations in serum levels of

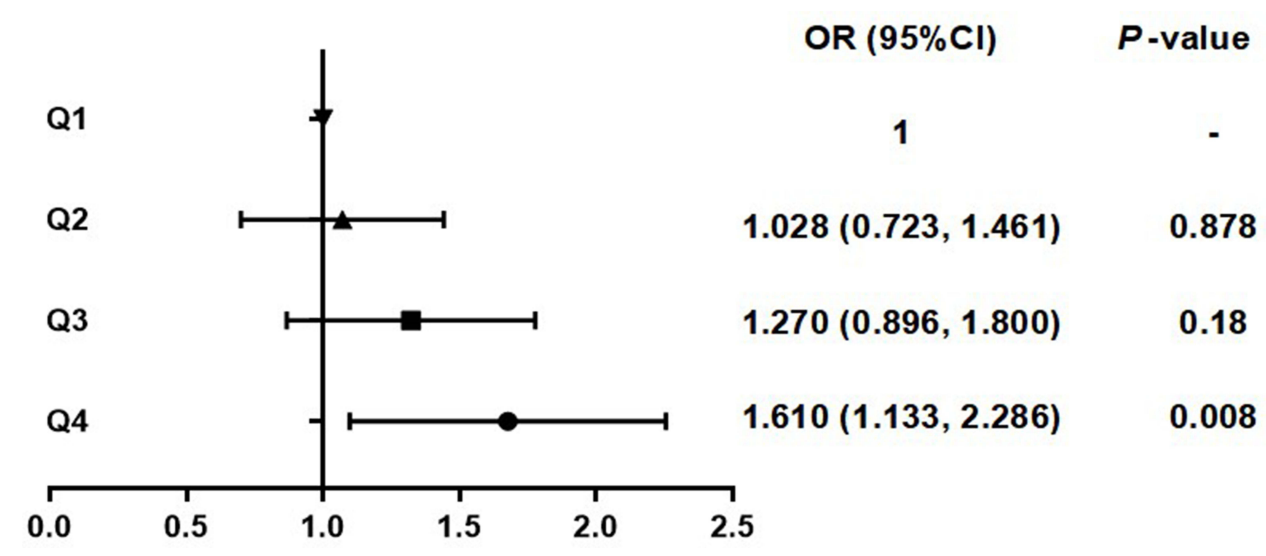

Figure 3 Logistic regression analysis regarding the effects of TSH level on the risk of NAFLD (Model 2). 


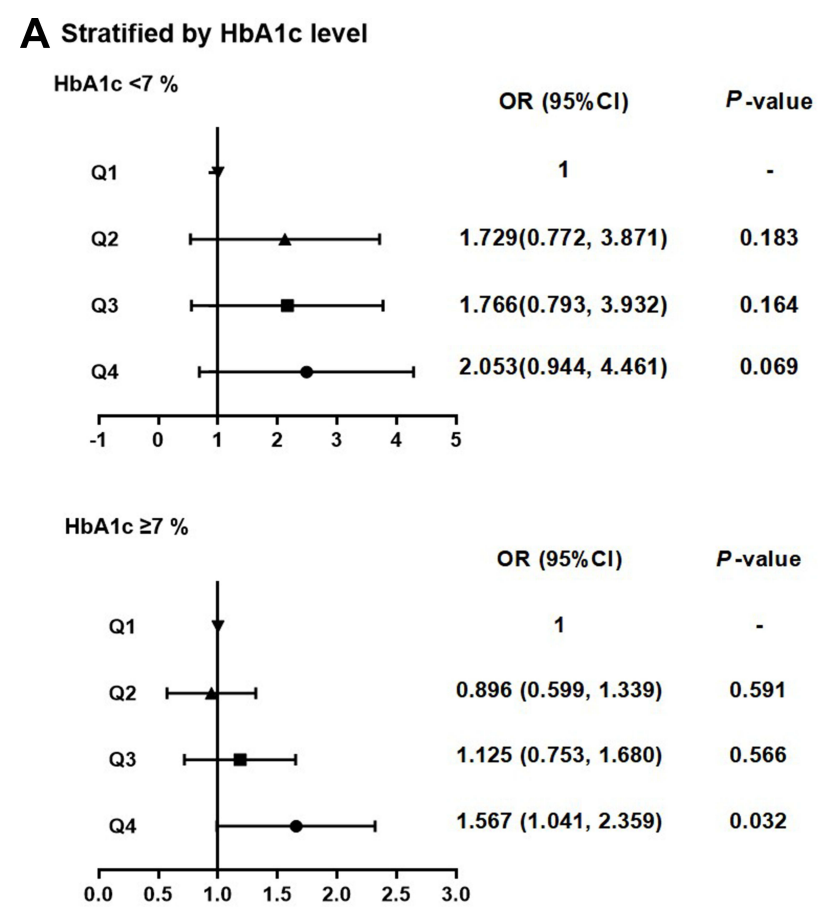

B Stratified by BMI level

$\mathrm{BMI}<28 \mathrm{~kg} / \mathrm{cm}^{2}$

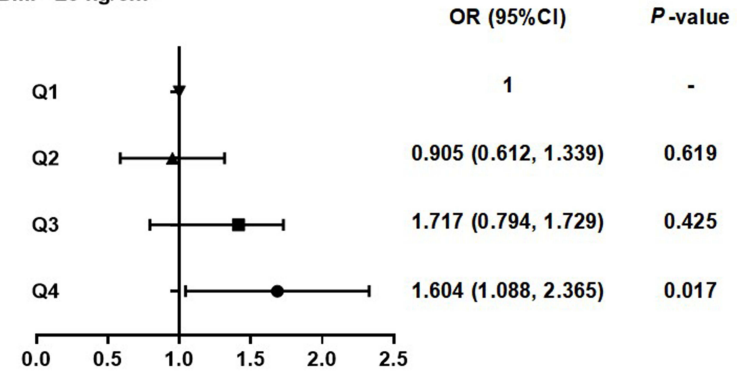

BMI $\geq 28 \mathrm{~kg} / \mathrm{cm}^{2}$

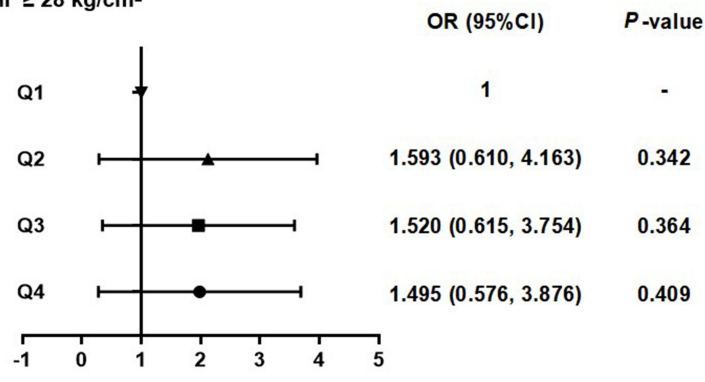

Figure 4 Subgroup analysis of the effects of TSH level on the risk of NAFLD (Model 2). (A) Subgrouped by HbAIC. (B) Subgrouped by BMI.

cytokines and adipokines will affect liver inflammation and increase lipogenesis. ${ }^{34}$ The level of TSH could affect the level of adipokines, thus contributing to the development of NAFLD. ${ }^{36,37}$

Our study has several limitations. The diagnosis of hepatic steatosis in our study was confirmed using ultrasonography but not liver biopsy, which is the gold standard for the diagnosis of NAFLD. ${ }^{14,38}$ Nevertheless, conventional ultrasonography, a non-invasive assessment, is the most commonly used imaging method for the diagnosis of hepatic steatosis because it is widely available, well established, well tolerated, and inexpensive. European guidelines for the management of NAFLD recommend using ultrasonography as first-choice imaging in adults at risk for NAFLD. ${ }^{39}$ Second, this is a cross-sectional study; therefore, this study could not reflect the causal effect of TSH levels on NAFLD in diabetic patients. Third, anti-thyroid peroxidase and anti-thyroglobulin autoantibodies were not considered in the present study. Thus, the possible influence of impending thyroid autoimmunity on the association between NAFLD and thyroid function cannot be ruled out.

\section{Conclusions}

In summary, our study demonstrated that a high-normal serum TSH level be significantly associated with the presence of NAFLD in type 2 diabetic patients with normal thyroid function. Thyroid hormone levels may need to be considered in evaluating the risk of NAFLD in T2DM and providing novel insight for treating NAFLD. More studies are needed to confirm the mechanism of TSH and NAFLD in diabetic patients with normal thyroid function.

\section{Statement of Ethics}

We promised that the data of the participants were anonymized or maintained with confidentiality and confirmed that the guide lines outlined in the Declaration of Helsinki were followed.

\section{Funding}

This study was funded by National Key R\&D Program of China (2017YFA0105803), the National Natural Science Foundation of China (81770826, 82000278), the 5010 Clinical Research Projects of Sun Yat-sen University (2015015), the Key Area R\&D Program of Guangdong Province (2019B020227003), the Science and Technology Plan Project of Guangzhou City(202007040003), the Guangdong Basic and Applied Basic Research Foundation (2020A1515010599), and the fostering special funding projects of the National Natural Science Foundation of China in the third affiliated hospital of SYSU(2020GZRPYQN04).

\section{Disclosure}

The authors report no conflicts of interest in this work. 


\section{References}

1. Rinella ME. Nonalcoholic fatty liver disease: a systematic review. JAMA. 2015;313(22):2263-2273. doi:10.1001/jama.2015.5370

2. Chalasani N, Z Younossi, JE Lavine, et al. The diagnosis and management of nonalcoholic fatty liver disease: practice guidance from the American Association for the Study of Liver Diseases. Hepatology. 2018;67(1):328-357.

3. Younossi ZM, Golabi P, de Avila L, et al. The global epidemiology of NAFLD and NASH in patients with type 2 diabetes: a systematic review and meta-analysis. $J$ Hepatol. 2019;71(4):793-801. doi:10.1016/j.jhep.2019.06.021

4. Byrne CD, Targher G. NAFLD: a multisystem disease. J Hepatol. 2015;62(1 Suppl):S47-64. doi:10.1016/j.jhep.2014.12.012

5. Bril F, Cusi K. Nonalcoholic fatty liver disease: the new complication of type 2 diabetes mellitus. Endocrinol Metab Clin North Am. 2016;45(4):765-781. doi:10.1016/j.ecl.2016.06.005

6. Mullur R, Liu YY, Brent GA. Thyroid hormone regulation of metabolism. Physiol Rev. 2014;94(2):355-382. doi:10.1152/ physrev.00030.2013

7. Sinha RA, Singh BK, Yen PM. Direct effects of thyroid hormones on hepatic lipid metabolism. Nat Rev Endocrinol. 2018;14(5):259-269. doi:10.1038/nrendo. 2018.10

8. Mantovani A, Nascimbeni F, Lonardo A, et al. Association between primary hypothyroidism and nonalcoholic fatty liver disease: a systematic Review and meta-analysis. Thyroid. 2018;28 (10):1270-1284. doi:10.1089/thy.2018.0257

9. Ludwig U, Holzner D, Denzer C, et al. Subclinical and clinical hypothyroidism and non-alcoholic fatty liver disease: a cross-sectional study of a random population sample aged 18 to 65 years. BMC Endocr Disord. 2015;15(1):41. doi:10.1186/s12902-015-0030-5

10. van den Berg EH, van Tienhoven-wind LJN, Amini M, et al. Higher free triiodothyronine is associated with non-alcoholic fatty liver disease in euthyroid subjects: the Lifelines Cohort Study. Metabolism. 2017;67:62-71. doi:10.1016/j.metabol.2016.11.002

11. Liu Y, Wang W, Yu X, et al. Thyroid function and risk of non-alcoholic fatty liver disease in euthyroid subjects. Ann Hepatol. 2018;17(5):779-788. doi:10.5604/01.3001.0012.3136

12. Kim D, Kim W, Joo SK, et al. Subclinical hypothyroidism and low-normal thyroid function are associated with nonalcoholic steatohepatitis and fibrosis. Clin Gastroenterol Hepatol. 2018;16(1):123131.e1. doi:10.1016/j.cgh.2017.08.014

13. Alberti KG, Zimmet PZ. Definition, diagnosis and classification of diabetes mellitus and its complications. Part 1: diagnosis and classification of diabetes mellitus provisional report of a WHO consultation. Diabet Med. 1998;15(7):539-553. doi:10.1002/(SICI) 1096-9136(199807) 15:7<539::AID-DIA668>3.0.CO;2-S

14. Fatty Liver Expert Committee, Chinese Medical Doctor Association. [Guidelines of prevention and treatment for nonalcoholic fatty liver disease: a 2018 update]. Zhonghua Gan Zang Bing Za Zhi. 2018;26 (3):195-203. doi:10.3760/cma.j.issn.1007-3418.2018.03.008 [Chinese]

15. Matthews DR, Hosker JP, Rudenski AS, et al. Homeostasis model assessment: insulin resistance and ?-cell function from fasting plasma glucose and insulin concentrations in man. Diabetologia. 1985;28 (7):412-419. doi:10.1007/BF00280883

16. Tilg H, Moschen AR, Roden M. NAFLD and diabetes mellitus. Nat Rev Gastroenterol Hepatol. 2017;14(1):32-42. doi:10.1038/ nrgastro.2016.147

17. Targher G, Bertolini L, Poli F, et al. Nonalcoholic fatty liver disease and risk of future cardiovascular events among type 2 diabetic patients. Diabetes. 2005;54(12):3541-3546. doi:10.2337/ diabetes.54.12.3541

18. Mantovani A, Zaza G, Byrne CD, et al. Nonalcoholic fatty liver disease increases risk of incident chronic kidney disease: a systematic review and meta-analysis. Metabolism. 2018;79:64-76. doi:10.1016/j.metabol.2017.11.003
19. Allen AM, Therneau TM, Larson JJ, et al. Nonalcoholic fatty liver disease incidence and impact on metabolic burden and death: a 20 year-community study. Hepatology. 2018;67(5):1726-1736. doi: $10.1002 /$ hep. 29546

20. Lonardo A, Bellentani S, Argo CK, et al. Epidemiological modifiers of non-alcoholic fatty liver disease: focus on high-risk groups. Dig Liver Dis. 2015;47(12):997-1006. doi:10.1016/j.dld.2015.08.004

21. Bruinstroop E, Dalan R, Cao Y, et al. Low-dose levothyroxine reduces intrahepatic lipid content in patients with type 2 diabetes mellitus and NAFLD. $J$ Clin Endocrinol Metab. 2018;103 (7):2698-2706. doi:10.1210/jc.2018-00475

22. Guo Z, Li M, Han B, et al. Association of non-alcoholic fatty liver disease with thyroid function: a systematic review and meta-analysis. Dig Liver Dis. 2018;50(11):1153-1162. doi:10.1016/j. dld.2018.08.012

23. Oda T, Taneichi H, Takahashi K, et al. Positive association of free triiodothyronine with pancreatic beta-cell function in people with prediabetes. Diabet Med. 2015;32(2):213-219. doi:10.1111/ dme. 12589

24. Garduno-Garcia JJ, Alvirde-Garcia U, López-Carrasco G, et al. TSH and free thyroxine concentrations are associated with differing metabolic markers in euthyroid subjects. Eur $J$ Endocrinol. 2010;163 (2):273-278. doi:10.1530/EJE-10-0312

25. Gu Y, Li H, Bao X, et al. The relationship between thyroid function and the prevalence of type 2 diabetes mellitus in euthyroid subjects. $J$ Clin Endocrinol Metab. 2017;102(2):434-442. doi:10.1210/ jc.2016-2965

26. Chung GE, Kim D, Kim W, et al. Non-alcoholic fatty liver disease across the spectrum of hypothyroidism. J Hepatol. 2012;57 (1):150-156. doi:10.1016/j.jhep.2012.02.027

27. Pagadala MR, Zein CO, Dasarathy S, et al. Prevalence of hypothyroidism in nonalcoholic fatty liver disease. Dig Dis Sci. 2012;57 (2):528-534. doi:10.1007/s10620-011-2006-2

28. Carulli L, Ballestri S, Lonardo A, et al. Is nonalcoholic steatohepatitis associated with a high-though-normal thyroid stimulating hormone level and lower cholesterol levels? Intern Emerg Med. 2013;8 (4):297-305. doi:10.1007/s11739-011-0609-4

29. Huang B, Yang S, Ye S. Association between thyroid function and nonalcoholic fatty liver disease in euthyroid type 2 diabetes patients. $J$ Diabetes Res. 2020;2020:6538208. doi:10.1155/2020/6538208

30. Jun JE, Jin S-M, Jee JH, et al. TSH increment and the risk of incident type 2 diabetes mellitus in euthyroid subjects. Endocrine. 2017;55 (3):944-953. doi:10.1007/s12020-016-1221-1

31. Su JB, Zhao L-H, Zhang X-L, et al. High-normal serum thyrotropin levels and increased glycemic variability in type 2 diabetic patients. Endocrine. 2018;61(1):68-75. doi:10.1007/s12020-018-1591-7

32. Giandalia A, Russo GT, Romeo EL, et al. Influence of high-normal serum TSH levels on major cardiovascular risk factors and visceral adiposity index in euthyroid type 2 diabetic subjects. Endocrine. 2014;47(1):152-160. doi:10.1007/s12020-013-0137-2

33. Hazlehurst JM, Woods C, Marjot T, et al. Non-alcoholic fatty liver disease and diabetes. Metabolism. 2016;65(8):1096-1108. doi:10.1016/j.metabol.2016.01.001

34. Musso G, Gambino R, Durazzo M, et al. Adipokines in NASH: postprandial lipid metabolism as a link between adiponectin and liver disease. Hepatology. 2005;42(5):1175-1183. doi:10.1002/hep.20896

35. Ortiga-Carvalho TM, Oliveira KJ, Soares BA, et al. The role of leptin in the regulation of TSH secretion in the fed state: in vivo and in vitro studies. $J$ Endocrinol. 2002;174(1):121-125. doi:10.1677/ joe. 0.1740121

36. Hui JM, Hodge A, Farrell GC, et al. Beyond insulin resistance in NASH: TNF-alpha or adiponectin? Hepatology. 2004;40(1):46-54. doi: $10.1002 /$ hep. 20280

37. Gong N, Gao C, Chen X, et al. Adipokine expression and endothelial function in subclinical hypothyroidism rats. Endocr Connect. 2018;7 (2):295-304. doi:10.1530/EC-18-0007 
38. Wong VW, Chan W-K, Chitturi S, et al. Asia-pacific working party on non-alcoholic fatty liver disease guidelines 2017-Part 1: definition, risk factors and assessment. $J$ Gastroenterol Hepatol. 2018;33 (1):70-85. doi:10.1111/jgh.13857
39. EASL E. EASL-EASD-EASO Clinical Practice Guidelines for the management of non-alcoholic fatty liver disease. J Hepatol. 2016;64 (6):1388-1402. doi:10.1016/j.jhep.2015.11.004

\section{Publish your work in this journal}

Diabetes, Metabolic Syndrome and Obesity: Targets and Therapy is an international, peer-reviewed open-access journal committed to the rapid publication of the latest laboratory and clinical findings in the fields of diabetes, metabolic syndrome and obesity research. Original research, review, case reports, hypothesis formation, expert opinion and commentaries are all considered for publication. The manuscript management system is completely online and includes a very quick and fair peer-review system, which is all easy to use. Visit http://www.dovepress.com/testimonials.php to read real quotes from published authors. 